\title{
A Wearable Exoskeleton Rehabilitation Device for Paralysis - A Comprehensive Study
}

\author{
Ahmed Roshdy, Samer Al Kork* , Sherif Said, Taha. Beyrouthy \\ College of Engineering and Technology, American University of the Middle East, Kuwait
}

\begin{tabular}{l} 
A R T I C L E I N F O \\
\hline Article history: \\
Received: 20 December, 2018 \\
Accepted: 27 December, 2018 \\
Online : 20 January, 2019
\end{tabular}

Keywords:

Rehabilitation

EEG

Prosthetic

Emotiv headset

Paralysis

\begin{abstract}
A B S T R A C T
As the technology grows scientists and engineers are trying to combine their work to compensate some body parts that is lost. Prosthetic devices grabbed the attention of most of the doctors and engineers working on solution for lost body parts. Generally prosthetic devices are either external wearable devices or internal ones. Such devices may depend on a built on microcontroller or the brain signals from the patient himself. Although it is used for lost body parts it can also be used for rehabilitation, power assistance, diagnostics, monitoring, ergonomics, etc. The currently used devices usually have the disadvantages of big size and high cost. The suggested device in this paper is targeting the design of portable rehabilitation device with light weight and low cost for paralyzed hand people. The suggested device allows the user to train the patient's hand or perform some needed exercises for his impaired hand. This helps the user to restore the normal hand movement and functionality. The device includes two modes of operation to be chosen by the user through the platform built on a microprocessor which can help controlling the exoskeleton to perform the needed exercises or tasks. Collaboration with several healthcare organizations will be considered to verify or test the effectiveness of this exoskeleton.
\end{abstract}

\section{Introduction}

This paper is an extension of work originally presented in BioSMART, the 2nd International Conference on Bioengineering for Smart Technologies" titled "A wearable rehabilitation device for paralysis' [1]. Diseases like stroke may lead to the loss of some parts of the human body and some other people are actually suffering of paralysis, broken bones, spinal cord injury, hemiplegia or traumatic brain injury. People facing such diseases or injuries are actually facing some problems in their day to day activities because of the infected or injured body part. One of the suggested solutions for such cases is the rehabilitation therapy to improve the infected part movement and regain the strength and power of that part. Some of the physiotherapy exercises are needed for the recovering rehabilitation process for the patient to be able to completely or partially restore the normal movement of the paralyzed body part [2]. Although this process is needed for hands or legs rehabilitation, this paper is actually focusing on a device to be used for the hands' problems and injuries. Humans are using their hands for writing, touching, holding, squeezing, and so many other functionalities. The partial or total loss of this part of the human body means losing so many valuable functions that any human being needs. The full hand with all its many joints act as natural motors to perform routine daily tasks [2]. This paper is meant to target a rehabilitation therapy system for patients with hand disabilities like too weak hands or even paralysis. The

*Samer Al Kork, American University of the Middle East, +965 22251400 Ext. 1732, samer.alkork@aum.edu.kw suggested device compared to the existing devices is considered to be low cost, lightweight, customizable, programmable and safe for human practice. All the functions of the device should be running in two different rehabilitation modes of training called active and passive. The design process will move into four phases, which are human interface, processing unit, health care sensors, and display. Each one of them has special task to do. Human interface will be responsible of the interface between the user and the processing unit. Processing unit will transmit commands to the electromechanical part. Electromechanical part will control the movement of the Exoskeleton. Health 10 care sensor will be used to monitor the user's health to insure the safety. The results will be shown in the display part.

The paper is going through literature review for common wearable devices available in the market showing the mode of control and the controlling method of each one. Also the literature review is covering the different brain waves' readers as well as the brain waves and the motor controls on the device itself. At the end the proposed wearable device is discussed mainly in hardware with a comparison to the available devices.

\section{Literature Review}

\subsection{Wearable Device}

Here in this section is the comparison between the main projects already exist as rehabilitation wearable devices and assistive exoskeletons. In Table 1 the comparison shows the difference in the modes and the methods used in some projects 


\section{A. Roshdy et al. / Advances in Science, Technology and Engineering Systems Journal Vol. 4, No. 1, 17-26 (2019)}

that were built for rehabilitation and assistive purposes for either hand or leg usage. Most of the projects are based in using either Virtual Reality (VR) or electromyography (EMG) as an interface for the device. None of the currently used devices has built the system using Electroencephalography (EEG) interface for the wearable device like what is offered in this study. The main problems for the devices already exist in the market are the high cost and the heavy weight because of the heavy materials used in their fabrication [4]. Although there already exist so many devices but only few of them offers the two modes of control passive and active. Herewith the study is based on EEG interface with the two possible modes of operation as well as keeping a light affordable weight and low cost.

Table 1 Some Applications of Wearable Devices

\begin{tabular}{|c|c|c|}
\hline Title & $\begin{array}{c}\text { Control } \\
\text { Modes }\end{array}$ & Methods \\
\hline $\begin{array}{c}\text { Design and development of a hand } \\
\text { exoskeleton for rehabilitation } \\
\text { following stroke [4] }\end{array}$ & Active & - \\
\hline $\begin{array}{c}\text { Hand Rehabilitation Support system } \\
\text { Based on Self-motion control, with } \\
\text { Clinical case report Error! Reference } \\
\text { source not found. }\end{array}$ & Active & VR \\
\hline $\begin{array}{c}\text { Current Hand Exoskeleton } \\
\text { Technologies for } \\
\text { Rehabilitation and Assistive } \\
\text { Engineering Error! Reference } \\
\text { source not found. }\end{array}$ & Passive & VR \\
\hline $\begin{array}{c}\text { Design and Development of a Hand } \\
\text { Exoskeleton Robot for } \\
\text { Active and Passive Rehabilitation [7] }\end{array}$ & Active/Passive & EMG \\
\hline $\begin{array}{c}\text { An EMG-driven exoskeleton hand } \\
\text { robotic training device on chronic } \\
\text { stroke [8] }\end{array}$ & Active & EMG \\
\hline $\begin{array}{c}\text { A review of technological and clinical } \\
\text { aspects of robot-aided rehabilitation } \\
\text { of upper-extremity after stroke [9] }\end{array}$ & Active & VR \\
\hline
\end{tabular}

Hong Kong polytechnic University designed a portable set of robotic hand exoskeleton that can be used or carried anywhere to help stroke patients in opening or closing their hands [8]. It works in an active mode and has 2 degree of freedom for each finger at the MCP and PIP. The robotic hand consists of Velcro straps to hold the hand in place, 5 linear actuators for fingers and a palm support platform. Finger assemblies are used to provide finger's flexion and extension. Also, it is designed in a way that can be used for different finger length. Also, there is an embedded controller that handles the robotic hand various tasks and monitor the EMG signals (sensor) that are used for the closing and opening process of a hand. Moreover, the set contains a wireless remote control system that helps the therapist to configure and select from different training modes (Figure 1).

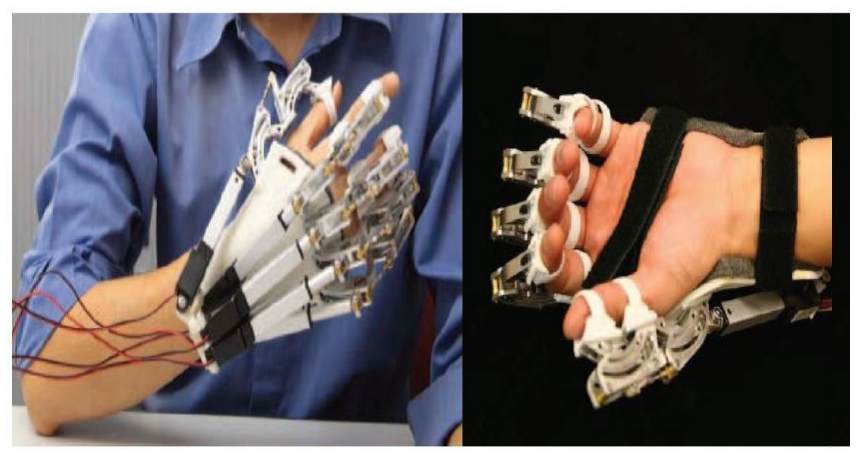

Figure 1. The Set of Hand Exoskeleton and Overview of the Prototype
University of Technology in Sydney Designed and developed a hand exoskeleton for rehabilitation following stroke [4]. The device is achieving full flexion/extension motion of the five fingers of the left hand (impaired hand) based on the motion of the identical digits of the right hand (healthy hand). The device is less than $2 \mathrm{Kg}$ and they chose Aluminum because of its lightweight. The hand exoskeleton has 15 degrees of freedom (DOFs). However, the hand exoskeleton cannot perform abduction/adduction movement; as a consequence, more work needs to be done on the device (Figure 2).

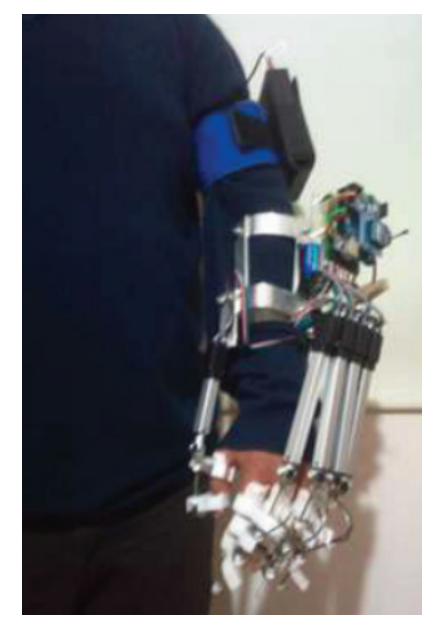

Figure 2. Hand Exoskeleton for Rehabilitation

Kyushu University proposed a hand exoskeleton using threelayered sliding spring mechanism [10]. The idea of this paper is to present a lightweight and compact device to easily use, so under-actuated mechanism is used to reduce the mass and size of the device by limiting the number of actuators into one (Figure 3 ).

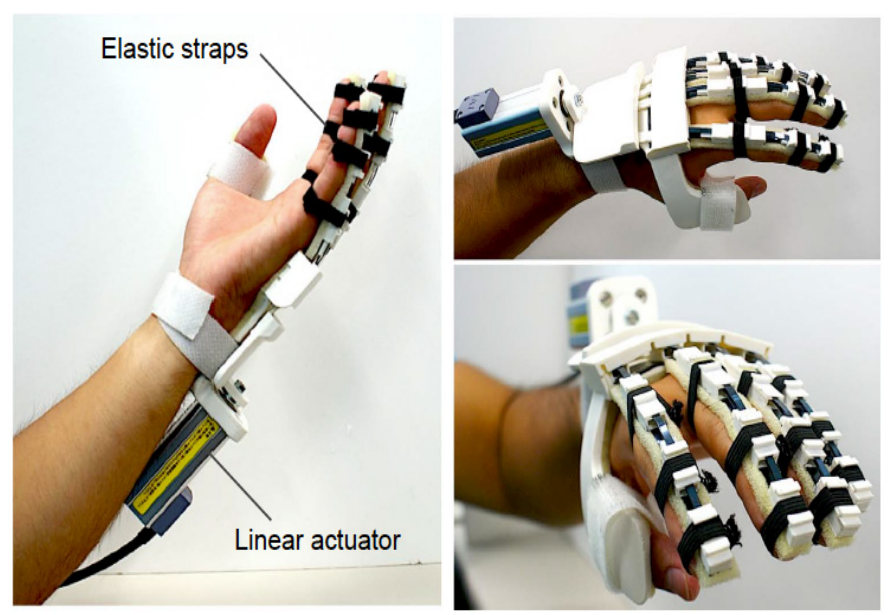

Figure 3. Overview of the Hand Exoskeleton

The weight of the exoskeleton is $320 \mathrm{~g}$. Each finger has three DOF, which is flexion/extension, but the thumb is fixed for the sake of robust grasping. Therefore, the three DOF is actuated through one actuator.as a result; the four fingers will work simultaneously. Three-layered sliding spring consists of 3 springs that is divided into inner $(\mathrm{Si})$, center $(\mathrm{Sc})$ and outer (So) springs and rigid bodies into tip (Rt), inner (ri) and outer (Ro) parts. The mechanism helps to perform flexion motion (Figure 4). 


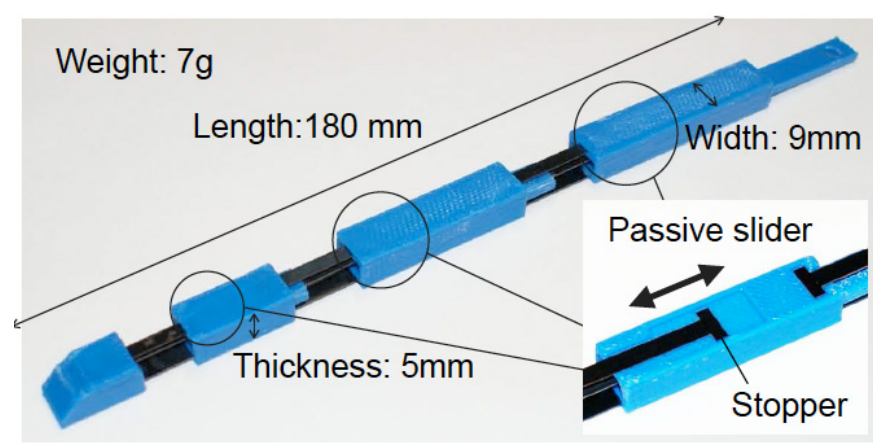

Figure 4. Three-Layered Sliding Spring Mechanism

University of Salford developed a hand assistive exoskeleton that operates in the active mode [11]. It uses virtual reality exerciser to perform the physical therapy exercises. It allows the patient to do the therapy exercises through fun interactive games. This exoskeleton allows hand motion, analysis and recording easily. The exoskeleton has 7 active DOF (Figure 5).

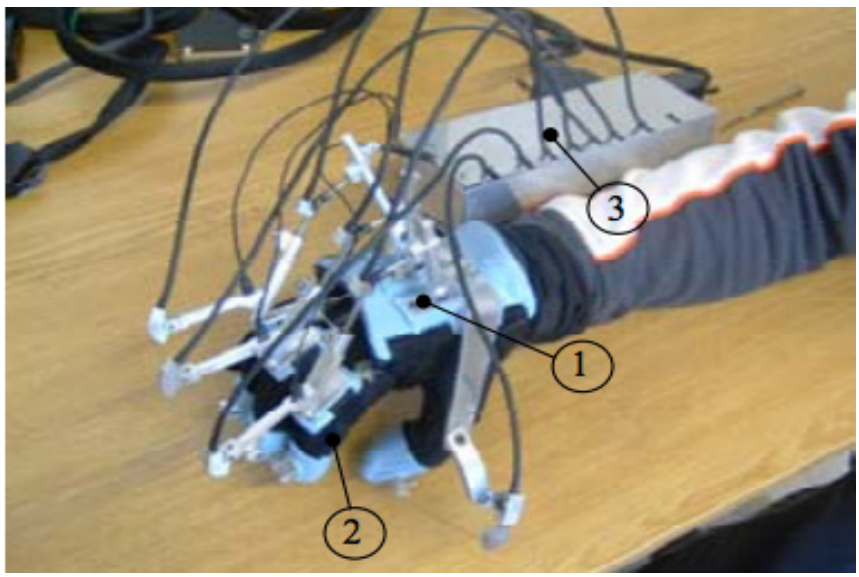

Figure 5. Assistive Exoskeleton

A team at Twenty university develop a low cost portable hand exoskeleton for assistive and rehabilitation in 2016 (Figure 6). They developed it to be for active rehabilitation, cheap, wearable, and portable [12]. This hand controlled by the mussels, they used EMG method. It can be for different sizes. They used $3 \mathrm{D}$ printer for some damages exoskeleton's components. The aim of this robotic hand exoskeleton is to assist persons with hand opening disabilities.

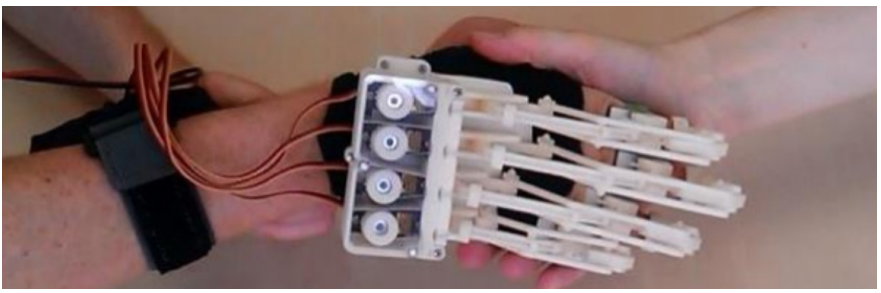

Figure 6. Assistive/Rehabilitation Exoskeleton

\section{2. $E E G$}

EEG stands for "Electroencephalography". EEG is a technique used to screen and record electrical signals produced inside the brain (Figure 7). There are two EEG techniques, which are non-invasive and invasive. In the non-invasive technique, electrodes are utilized to record the signals, and they are attached directly to the human head. On the other hand, the invasive technique records the signals by embedding electrodes inside the skull itself till the brain. In addition, there are two different types of communication, the first one is the BCI (brain computer interface), and the second one is BMI (brain machine interface). These two types define the communication method or interface between the brain and the device meant to record the brain signals. Relating the mentioned two communication methods to EEG techniques the $\mathrm{BCI}$ communication type follows the non-invasive technique while the BMI type follows the invasive method. Using the EEG will allow the user to control the motors by his own brain signals. The brain signal captured by the EEG is to be sent to set of motors to perform the needed movement or action. These days, this technique has turned out to be affordable and available to general society. Many different products already available in the market are using EEG in order to monitor the brain activity.

\section{EEG Signal Collection}

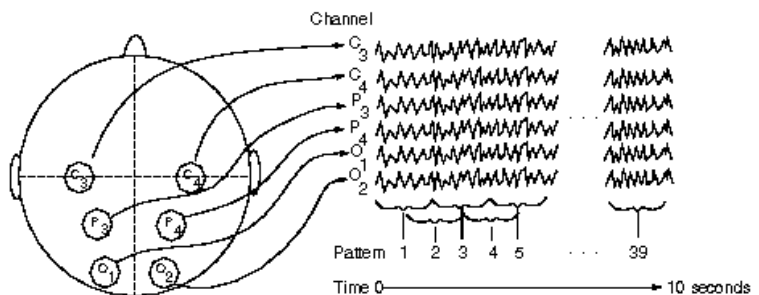

Figure. 7. EEG electrodes record signals from brain

The brain is a very complex and essential organ that its main function is to control the whole body. In general, the brain anatomy or structure is consisting of three main sections these are forebrain, midbrain and hindbrain. The forebrain is classified into three parts, which are thalamus, cerebrum, and hypothalamus. The cerebrum represents the largest part of the brain. It is divided into 4 portions called lobes, which are frontal $(\mathrm{F})$, parietal $(\mathrm{P})$, temporal $(\mathrm{T})$, and occipital $(\mathrm{O})$ lobes.

Each area or region has its own function. The frontal lobe is responsible for problem solving, speaking, judgment, emotional expression, thinking, planning and movement.

Parietal lobe is more into processing sensory and interpreting visual information (reacting into environment); it allows sensation from muscle and skin, body orientation, and reading. Temporal lobe is associated with behavior, memory, hearing and understanding language. Moreover, the occipital lobe is used for color perception, image recognition and vision or sight (Figure 8).

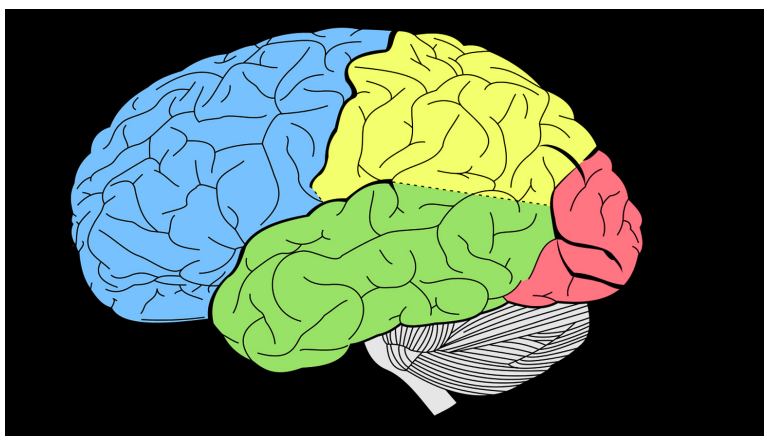

Figure 7. The Main Portions of the Brain 


\subsection{Emotiv (EPOC+) and the 10-20 System}

EPOC follows the 10-20 system electrode placements which is considered to be an international system [13]. This system depends on $10 \%$ or $20 \%$ separation between the electrodes. It permits 21 electrodes on the surface of the scalp shown in Figure.. Each possible location for the electrodes is described by a letter to identify the lobe and a number to define the location of the hemisphere (left or right side of the brain). It shows the 14 channels of EPOC are distributed as frontal (AF3, AF4, F7, F3, F4, F4), front-central (FC5, FC6), parietal (P8, P7), temporal (T8, T7) and occipital (O1, O2) lobes [14]. The odd numbers refer to the left side of the brain and the even numbers refer to the right side of the brain. The system renamed some locations or points, which are T4, T3, T5 and T6 to be T8, T7, P7 and P8 respectively. Also, the AF and FC are intermediate sites; AF is between Fp and $\mathrm{F}$ but $\mathrm{FC}$ is between $\mathrm{F}$ and $\mathrm{C}$. Moreover, $\mathrm{A} 1$ and $\mathrm{A} 2$ are the locations of earlobes, DRL and CMS correspond to P3 and P4, which are the reference sensors (Figure 9).

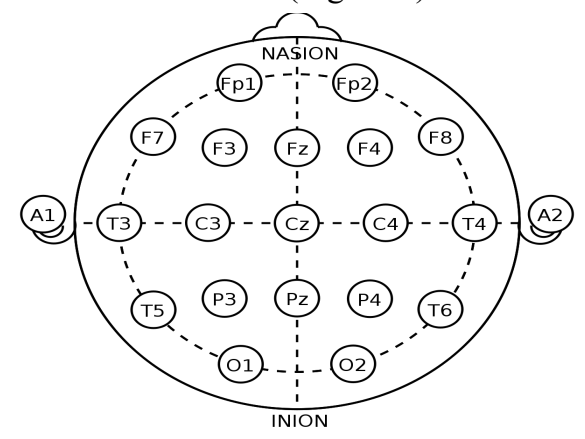

Figure.9. Locations of the reference sensors

\subsection{The Motor Area}

As the different brain parts are generating different types of waves for each task needs to be performed by the human body, the headset. The primary motor cortex, which is located in the frontal lobe, is responsible for controlling the execution of movement [15]. In fact, it is located in an area called Precentral gyrus. This part of the brain is participating in controlling the movement of different parts of the body like arm, hand, face, foot, etc. (Figure 10). According to the 10-20 system brain map, C3, Cz and $\mathrm{C} 4$ are the nearest to the location where motor execution occurs [15], [16]. Unfortunately, the three motor locations aren't available in the EPOC headset. However, there is an easy way to obtain one of the three points; the headset can be tilted a little bit till pointing one of the electrodes to one of the needed three locations.

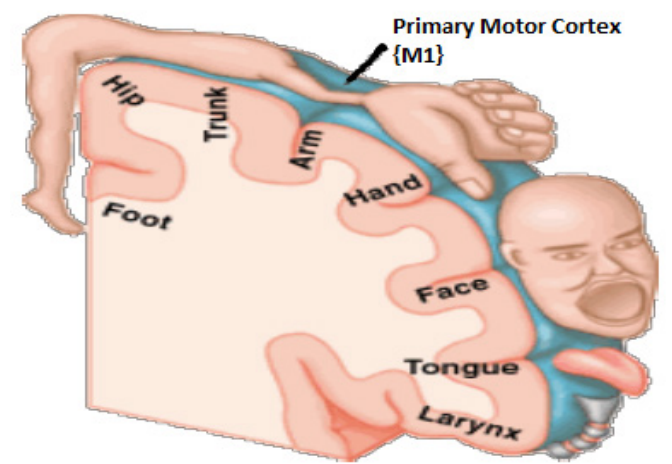

Figure 10. The Location of the Hand Movement in the Motor Cortex

\subsection{Brainwaves}

The brain signals are different according to the band of frequencies of each one of them. The different frequencies or to be more precise the different band of frequencies are covering all the brain activities whether the human body is moving or not. Each wave generated by the brain is does have particular location to be produced from. The main 5 range of frequencies from the brain are Delta, Beta, Theta, Alpha, and Gamma (Figure 11). Each band has a specific frequency range but a different meaning.

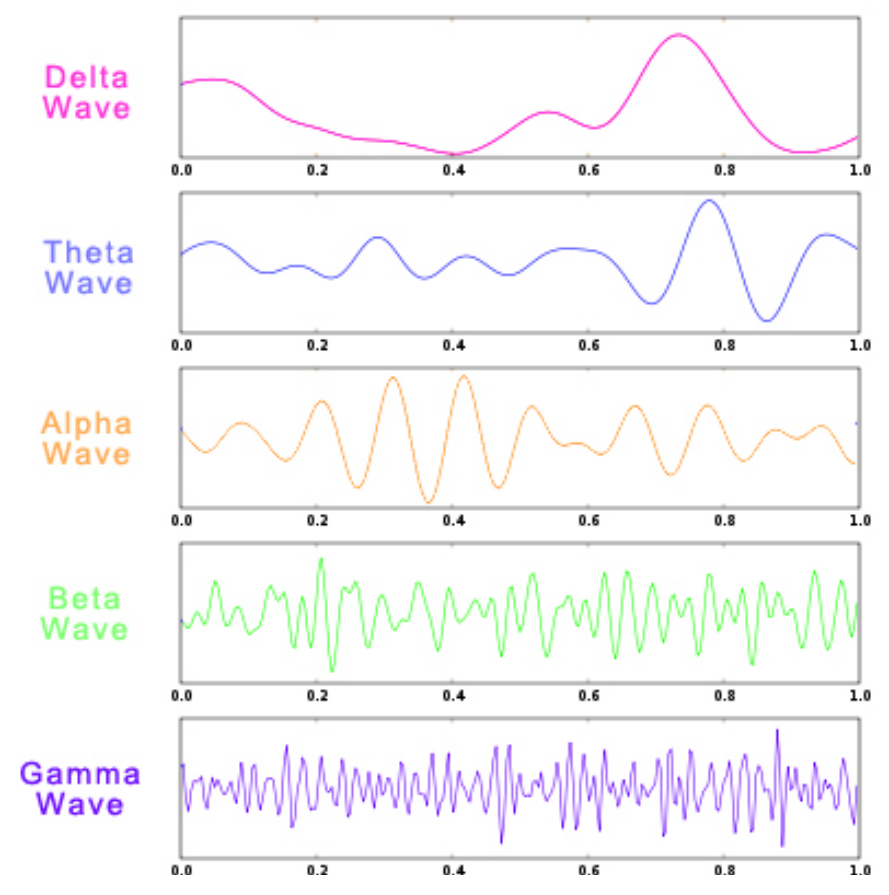

Figure 11. The Main 5 Brainwaves

The first range of frequencies is from 0.5 to $3 \mathrm{~Hz}$ and considered to be the Delta range. This range of waves are created while sleeping or relaxing meditation sessions. Also in our scope of work the Delta waves are generated during the normal human body healing during a deep sleep. The next range of waves is the Theta waves and it's in the range of $4-8 \mathrm{~Hz}$. Usually these kind of waves generated while sleeping as well or laziness. Third range for the Alpha waves is from 4 to $12 \mathrm{~Hz}$ and the got generated by the occipital, parietal and frontal lobes in some cases like closing the eyes and by the frontal lobe during relaxation. Out of the Alpha waves range there is a range from $8-12 \mathrm{~Hz}$ is called $\mathrm{Mu}$ waves. The $\mathrm{Mu}$ waves mainly corresponds to the body movement. In other words, the Mu waves are generated when the human body is relaxed. The next range from 12 to $25 \mathrm{~Hz}$ is called Beta waves. Beta waves are generally created in body movements and brain activities like thinking or problem solving. Above $25 \mathrm{~Hz}$ signals are called Gamma signals and they are faster than the other waves as noticed from its frequency. Such signals are usually generated with excessive brain work during multi-tasking.

\subsection{Virtual Reality}

Virtual reality represents an alternative way to be engaged or involved in the rehabilitation therapy routines and not easily get bored by using the rehabilitation gaming system [18],[19]. The rehabilitation gaming system is a virtual reality tool in a three- 


\section{A. Roshdy et al. / Advances in Science, Technology and Engineering Systems Journal Vol. 4, No. 1, 17-26 (2019)}

dimensional world that let the patient does the therapy exercises through fun interactive games (Figure 12). Also, it can be used as a stimulus to help the patient in the motor imagery process. The VR technology is currently a very up-to-date trend. It is quickly moving towards the mainstream that even big companies are investing in this technology like Samsung, Sony, Apple, etc. They are developing their existing devices to follow this new technology.

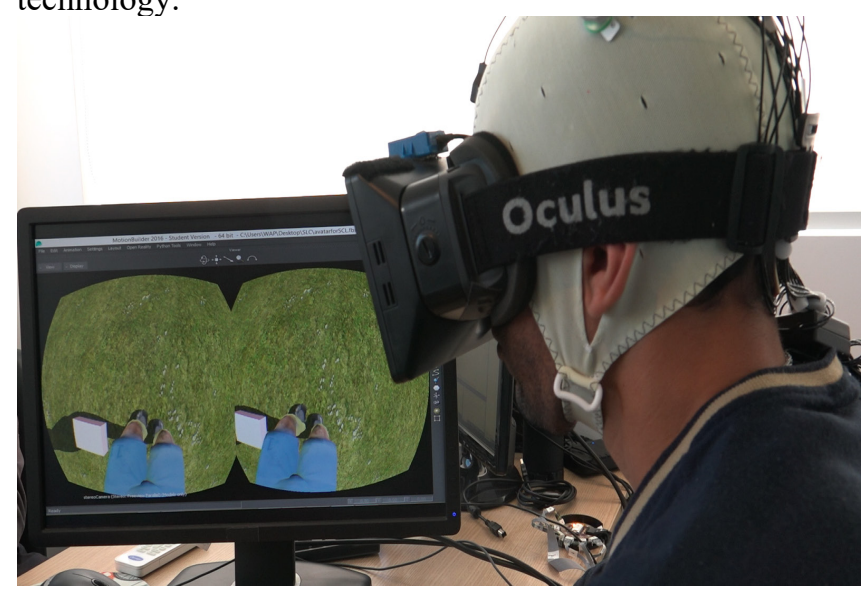

Figure 12. Virtual Reality Tool in a Three-Dimensional World

\subsection{Electromyography (EMG)}

Electromyography EMG measures electrical current inside muscles (Figure 13). EMG is controlled by nervous system. It gains noise while moving through different tissues. Specific Algorithms should be used to reduce the noise in order to obtain accurate EMG signal [20]. Many applications have been implemented using EMG method for exoskeleton hand control; they used muscle signals to control the impaired hand which is driven by an intact organ [8].

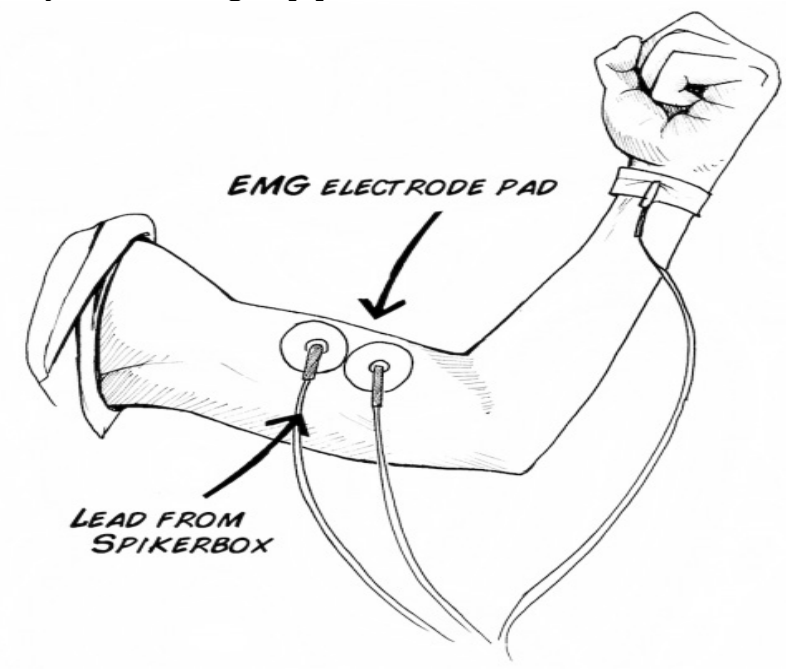

Figure 13. EMG Signals Comes From Muscles

\section{Detailed design}

\subsection{Design Overview}

The whole design is described in Figure 18 showing the whole design different stages through the design. In the active mode the EEG headset starts to read the brain signals and detects the location of the signal and decide the lobe and the side of the brain sending the signal. Next step the headset will wirelessly communicate with the computer through Wi-Fi to start the analysis. After the analysis is done the signal should be compared to previously recorded data to indicate the meaning of the brain signal based on pattern recognition. In the Preprocessing unit, the signal will be filtered. Then the signal goes to the second part where features and specific characteristic will be detected. The final stage the signal will go through is the classification where the imagined movement will be identified and performed. After signal processing, the information taken from the headset will be compared to the previous saved data. Using pattern recognition if the comparison got a match, then it will send the data through Bluetooth ZigBee module to the electromechanical parts to start the movement. This part will then give the instructions to the servo motors to start performing the needed movement. This part includes two big servos that ensure the movement of the arm up and down (Figure 14).
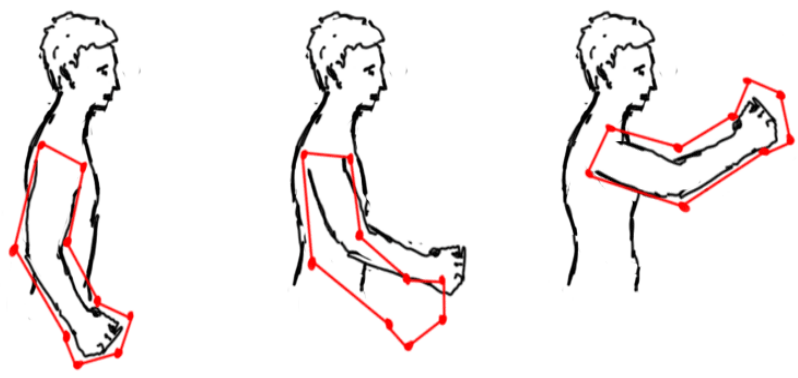

Figure 14. The movement of the arm

The health sensors part includes a body temperature sensor, which allows the user to measure his body temperature [20]. It includes also a pulse oximetry sensor that indicates the arterial oxygen saturation of functional hemoglobin. An LCD screen is present to show the results of some sensors like the body temperature sensor and it is considered to be a major part of the interface between the user and the device. Wi-Fi is used to send all the results to the mobile application. These results will lead to activating or deactivating the mechanical parts (motors). The schematic of the Robotic-Based Rehabilitation system with all its basic parts is shown in Figure 15.

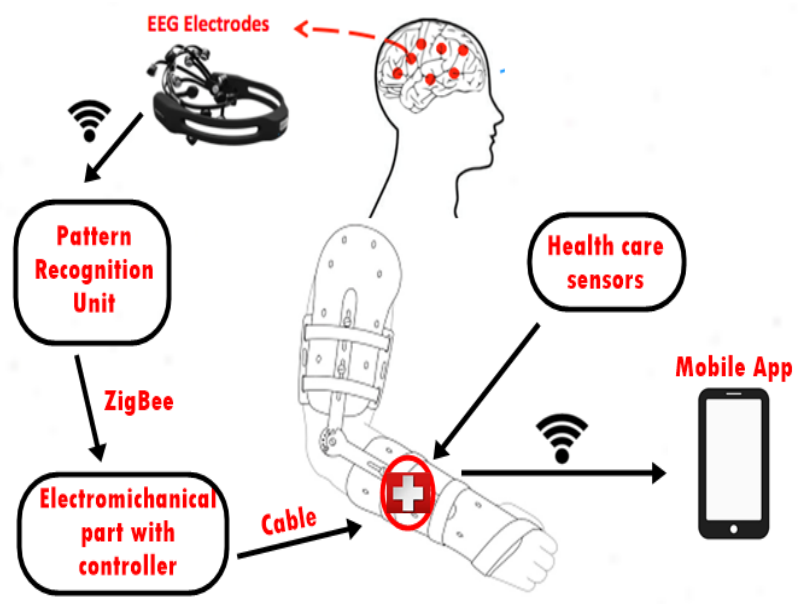

Figure 15. Design overview 


\section{A. Roshdy et al. / Advances in Science, Technology and Engineering Systems Journal Vol. 4, No. 1, 17-26 (2019)}

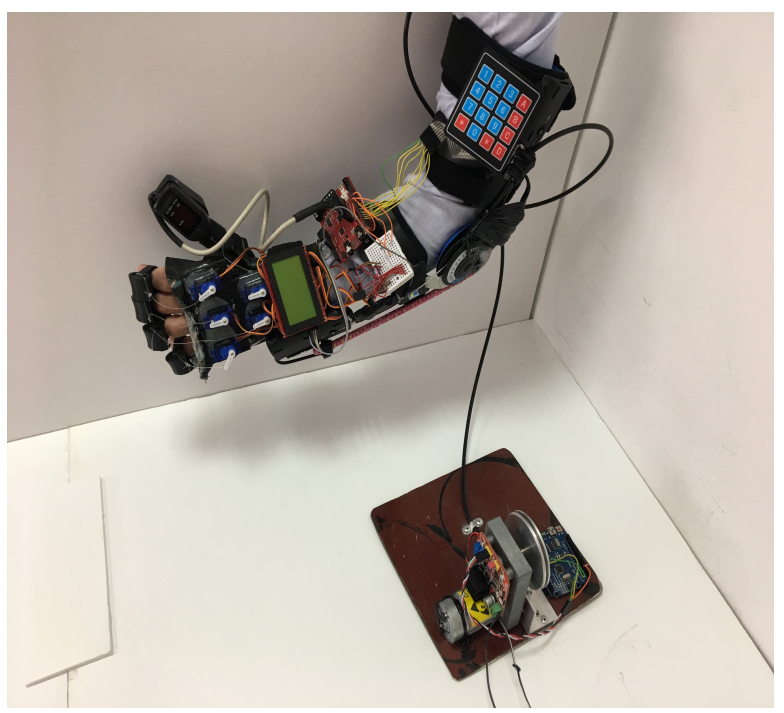

Figure 16. Design schematic

\subsection{System Design}

The overall design and flowchart is represented in Figure 16 and Figure 17. The user enters his/her age. Then, the temperature, ECG, and Pulses of the user are displayed on the LCD. If the values are in the normal ranges, the system continues running, otherwise, it stops. There are specific ranges for each one of them. The normal body temperature tends to be between the range $36.1^{\circ} \mathrm{C}$ to $37.2^{\circ} \mathrm{C}$. On the other hand, the normal human pulses can be classified according the ages to be 40 to 60 pulses for ages between 1 to 10 years and 60 to 100 pulses for ages between 10 to 60 years. Therefore, these things will provide extra security for the user.

Second, the user picks the required mode through the built on switch (left for active, middle for off, right for passive). If the user selected the first mode which is the passive mode, the user will be able to select the needed exercise through the keypad connected to the Arduino microcontroller. After that, the servo motors will start performing the corresponding movements based on the code on the Arduino board. The motors are connected through rods to the wearable device parts like fingers and rest to perform the right movement.

The other mode of operation is the Active mode that can be selected by moving the switch to the left. In the Active mode the movements and therapy will be controlled by the brain signals read by the EEG headset. When the headset reads the brain signal it will pass it to the Arduino for analyzing to recognize the required move Figure 18. In the beginning the signals are filtered whenever read by the EEG to get rid of the noise signals through bandpass filter and notch filter used particularly for $50 \mathrm{~Hz}$ signals that will be there most of the time from the surroundings and power sources around the patient [14]. After having the clean signal, the next step is getting the information from the signal and translate it into movements or exercise through the motors connected to the wearable device. The signals movement translated orders are saved in a features vector to be performed. The extracted information is classified in order to help in extracting the features into spatial and spectral information. The location of the electrode extracting the signal is considered to be spectral type of information. On the other hand, spectral information represents the power of the frequency bands. At the end a computer will classify the signals to be able to select the movement. There are many classifiers; however, neural network and SVM are most commonly used [23][24]],[14],[26]. The classifier will then save the obtained data in the feature vector with either movement to class A or class B. Comparing these data with the database stored from pre imagined movements will lead in case of matching to moving the signal to Arduino as the brain for the servo motors to perform the exercise and move the device.

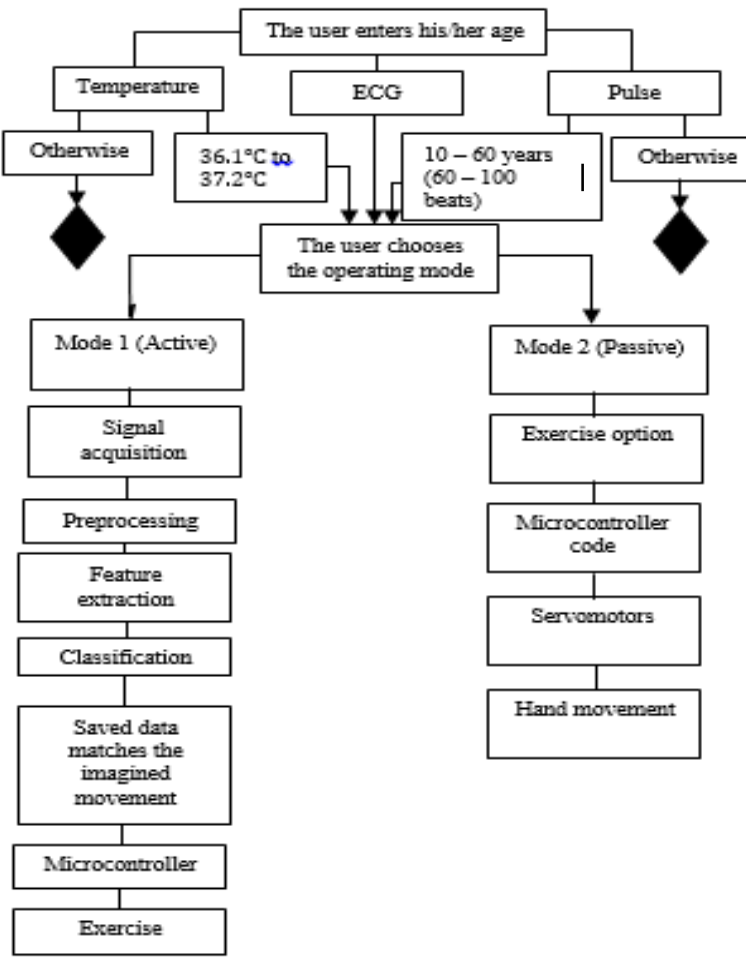

Figure 17. Flowchart of the system design

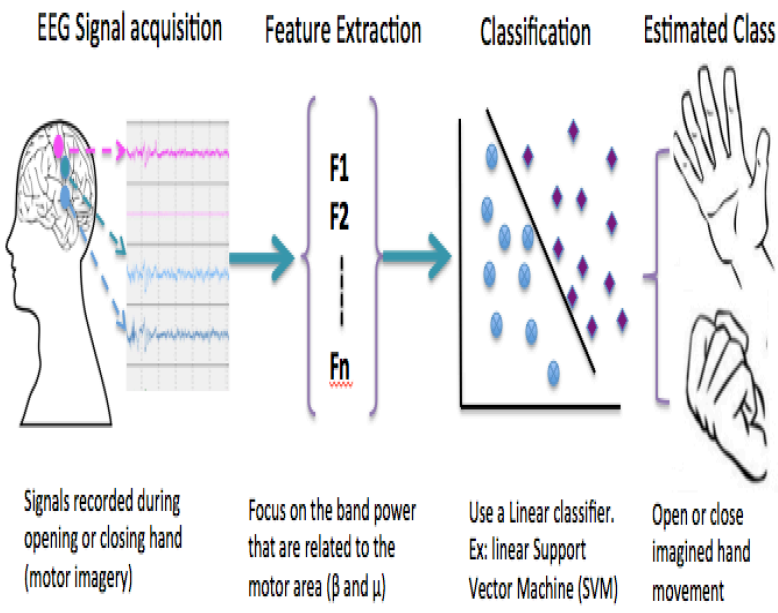

Figure 18. Signal processing steps [21][24]

\subsection{Relevant Engineering Application and Calculations}

Servomotors was chosen for this project. Four servo motors in two separate joints are needed for each arm. To check the loads 


\section{A. Roshdy et al. / Advances in Science, Technology and Engineering Systems Journal Vol. 4, No. 1, 17-26 (2019)}

on the motors and make sure about the safety of the human body, it was designed to allow the device to hold weights up to $10 \mathrm{~kg}$. This amount of weight causes the torque on the servo motors while moving. The angle of movement should also be taken into considerations to have the right calculations of the motors mechanical loads. The torque on the servomotors according to the applied force will be calculated using equation (1)

$$
\tau=F \times r \times \sin \emptyset
$$

Where $\tau$ is the applied torque, $F$ is the force cause by the weight carried $(F=m \times g), r$ is the distance, and $\varnothing$ is the maximum angle of movement which is $90^{\circ}$. The distance used in our design is $0.74 \mathrm{~m}$ and the mass of the arm is $3.628 \mathrm{~kg}$, and the gravity in Kuwait is 9.793N/A. The resulting torque is $26.29 \mathrm{~N}$.m. For such torque and safety, the servomotors will be chosen accordingly. From equation (1) there is a direct proportional relation between the torque and the applied force. Taking into considerations the power consumption, the no load power can be calculated using equation 2 knowing that the maximum current used with no load is $500 \mathrm{Ma}$.

$$
P=V \times I=25 \times 0.5=12 w
$$

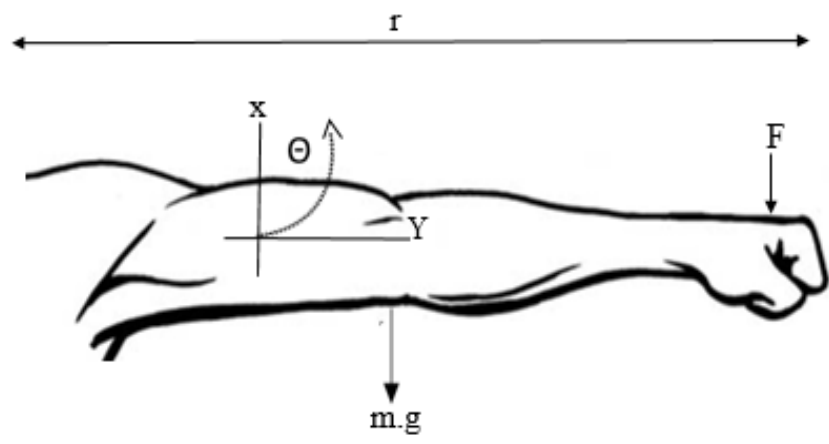

Figure 19. Power Consumption Parameters

\subsection{Materials}

For the process of building the hand exoskeleton device, so many materials need to be studied in terms of weight, safety, and flexibility in order to choose the suitable one for the project. Iron, aluminum and 3D printed material which is mainly plastic-based are different materials that can be used for exoskeleton. Each one of them has different properties. Iron is heavier than aluminum because of its density, which is $7.1 \mathrm{~g} / \mathrm{cc}$. Moreover, Iron is cheaper than aluminum because it does not need many processes to obtain as the aluminum. It is stronger than aluminum. Iron's strength is $169(\mathrm{MPa})$ whereas aluminum is $80(\mathrm{MPa})$. Aluminum takes time to be manufactured. The features of $3 \mathrm{D}$ printer materials are: cheap, flexible, durable, lightweight and available with varies colors. Easy to redesign and model if needs to do any changes. For example; if something is broken we can have reprinted it easily. Furthermore, it is not taking much time to print. The materials that should be used must be environment friendly to provide safety for a long period time. Aluminum is not environment friendly. The releasing of perfluorocarbons during the aluminum smelting process are 9,200 times more harmful than carbon dioxide. Iron is not recommended due to the chemicals which enters into the environment and affect the eco balance, and cause so many health problems. 3D printer materials like Acrylonitrile Butadiene Styrene (ABS) and Polylatic Acid (PLA) can be considered as environment friendly materials because it is easy to recycle, no waste of materials, and no more unsold product. The comparison is summarized in Table $2 \&$

Table 3

Table 2. Materials' Specifications

\begin{tabular}{|c|c|c|c|c|}
\hline Materials & $\begin{array}{c}\text { Price per } \\
\mathbf{k g} \mathbf{( \$ )}\end{array}$ & $\begin{array}{c}\text { Strength } \\
\text { (Mpa) }\end{array}$ & Density & $\begin{array}{c}\text { How easy to } \\
\text { customize }\end{array}$ \\
\hline Iron & $\begin{array}{c}0.049- \\
0.098\end{array}$ & 169 & $7.1 \mathrm{~g} / \mathrm{cc}$ & Difficult \\
\hline Aluminum & $\begin{array}{c}0.307- \\
1.221\end{array}$ & 80 & $2.68 \mathrm{~g} / \mathrm{cc}$ & Difficult \\
\hline ABS & 29.30 & $25-50$ & $\begin{array}{c}1.01-1.21 \\
\mathrm{Mg} / \mathrm{m}^{3}\end{array}$ & Easy \\
\hline PLA & 29.30 & $36-55$ & $\begin{array}{c}1.25 \mathrm{Mg} / \\
\mathrm{m}^{3}\end{array}$ & Easy \\
\hline
\end{tabular}

\begin{tabular}{|c|c|c|}
\hline Materials & Advantages & Disadvantages \\
\hline Iron & $\begin{array}{ll} & \text { Strong } \\
- & \text { Durable }\end{array}$ & $\begin{array}{ll} & \text { Heavy Weight } \\
\text { - } & \text { Expensive } \\
\text { - } & \text { Hard to recycle } \\
\text { - } & \text { Not environment } \\
& \text { friendly } \\
\text { - } & \text { Rusts } \\
\end{array}$ \\
\hline Aluminum & $\begin{array}{ll}\text { - } & \text { Light Weight } \\
\text { - } & \text { Durable } \\
\text { - } & \text { Won't Rust } \\
\text { - } & \text { Doesn't deteriorate }\end{array}$ & $\begin{array}{ll}\text { - } & \text { Not very strong } \\
\text { - } & \text { Expensive } \\
\text { - } & \text { Hard to recycle } \\
\text { - } & \text { Not environment } \\
& \text { friendly } \\
\text { - } & \text { Can easily be water } \\
& \text { stained }\end{array}$ \\
\hline $\begin{array}{l}\text { 3D Printer } \\
\text { Materials }\end{array}$ & $\begin{array}{l}\text { - Light Weight } \\
\text { - } \text { Cheap } \\
\text { - Easy to recycle } \\
\text { - Environment Friendly } \\
\text { - Flexible } \\
\text { - Durable } \\
\text { - Won't Rust } \\
\text { - Doesn't deteriorate }\end{array}$ & - Not very strong \\
\hline
\end{tabular}

Table 3. Advantages \& Disadvantages Comparison

\subsection{Electromechanical System}

1) Battery:

For a battery, there are different kinds of batteries and each of them has its own features. First of them is Lithium ion Battery which has very high capacity, normal size, long life, high cost and it is rechargeable. Second one is NiMH which has a small size, low capacity, short life, low cost and non-rechargeable.

Third one is NiCd has big size, low capacity, short life, low cost, and rechargeable. Final one is SLA-6v20 has very big size, very high capacity, long life, high cost, and rechargeable. Energizer NH15BP and Duracell have short life and low capacity so they will not benefit this project well. Lithium ion and SLA6 v20 both are good, but we will choose Lithium ion because its size is smaller. Batteries comparison shown in Table 4.

\section{2) Actuators:}

Actuators are mechanical devices that move or turn energy into motion. There are different types of actuators, which are electric 


\section{A. Roshdy et al. / Advances in Science, Technology and Engineering Systems Journal Vol. 4, No. 1, 17-26 (2019)}

actuators, hydraulic and pneumatic actuators. Electric actuators transfer electrical energy into mechanical energy. There are two types of electric actuators which are direct current motor (DC motor) and servomotors. Brushless DC motor is a type of DC motor that has different properties. It is small in size and weight not to mention its high speed and torque. On the other hand, a servomotor is a type of actuator that its functionality is to control motion. It operates within the limit of the specified angle (angular precision) by receiving a control signal to take further action. Moreover, it uses closed-loop feedback to control speed, torque or position. Pneumatic actuator is a hollow cylinder inside it a piston. The piston moves by applying a pressure from a pneumatic pump (compresses air) to create a force. Hydraulic actuators work similarly to Pneumatic actuators; except for the fact they are driven by liquid (fluid) pressure instead of air pressure. Pneumatic actuators are more expensive than hydraulic actuators. Moreover, hydraulic actuators are stronger or have greater force to move heavy loads.

Table 4. Batteries Types

\begin{tabular}{|c|c|c|c|c|c|}
\hline \multirow{2}{*}{ Specifications } & \multicolumn{3}{|c|}{ Li-Ion } & NiMH & NiCd \\
\cline { 2 - 6 } & Cobalt & Phosphate & Manganese & & \\
\hline $\begin{array}{c}\text { Energy } \\
\text { (Wh/Kg) }\end{array}$ & $150-$ & $100-150$ & $90-120$ & $60-120$ & $45-80$ \\
\hline $\begin{array}{c}\text { Life Cycle } \\
\text { (80\% DoD) }\end{array}$ & $500-$ & $500-1000$ & $1000-2000$ & $300-$ & $1000^{3}$ \\
\hline $\begin{array}{c}\text { Charge Time } \\
\text { (H) }\end{array}$ & $2-4$ & $1-2$ & $1-2$ & $2-4$ & $1-2$ \\
\hline Cell Voltage(v) & 14.8 & 3.7 & $3.2-3.3$ & 1.2 & 1.2 \\
\hline $\begin{array}{c}\text { Safety } \\
\text { Requirements }\end{array}$ & \multicolumn{7}{|c|}{ Protection circuit mandatory } & $\begin{array}{c}\text { Fuse protection, } \\
\text { thermally stable }\end{array}$ \\
\hline Cost & \multicolumn{7}{|c|}{ High } & \multicolumn{2}{c|}{ Moderate } \\
\hline
\end{tabular}

\subsection{Programming}

\section{3) Processor}

Nowadays, the advancement in the processors and controller's technology has made it affordable and possible to achieve most of project requirements. In today's market, the top leading and the most common three processors/controllers are Raspberry pi, Arduino and Intel Edison. Raspberry pi considers as a tiny computer that has its own operating system. It is Linux based operating system and it can multitask. The board includes a processor, Ram, USB ports and everything a normal computer has. There are four models or generations of raspberry pi, which are Raspberry Pi 1, Raspberry Pi 2, Raspberry Pi 3, and Raspberry zero. Each one of them has different properties and using them depend on the needs. In general, a raspberry pi allows the user to not be restricted or limited to a certain programing language. Also, it is very fast in processing and easy to connect to Internet

On the other hand, Arduino is a microcontroller that is consists of software and hardware. It is a board that contains a chip to be programmed and use it to perform several functions. It is flexible that can interact with different devices like LEDs, buttons, speakers, motors, and cameras. For instance, it can read data from sensors, get to blink an LED, etc. There are several types of Arduino but the most popular one is the Arduino UNO. Most Arduinos have the same components, which are a power source, a processor, digital and analog pins, a reset button and a USB port. Arduino is easier to work with and it is better in controlling than Raspberry pi. It is capable of controlling complex external hardware. Also, it is cheaper than Raspberry pi. There is also another type of microprocessor that considers as a competitor to raspberry pi. It is offered by Intel Company, which is called Intel Edison. It functions as a computer. The board has a processor, RAM, USB port, Bluetooth and WiFi. It is a little bit similar to Raspberry pi but each one of them has its own features. For example, Intel Edison is more expensive than raspberry pi. Also, it has less numbers of USB ports and the processor speed is lower than the one in raspberry pi.

On a separate note, microcontrollers and microprocessors show different weaknesses and strengths depending on the application. There is a difference between microcontroller and microprocessor and each has advantages and disadvantages. In general, microcontrollers are more suitable for controlling devices (such as servos, etc.) whereas the microprocessors are better in processing

\section{4) Interface}

There are so many user interfaces to help the user interact with the computer to detect and analyze specific information, or to get some feedback from it. EEG is one of the user interfaces that measured by using electrodes that is placed on the scalp. It can be recorded by the electric fields that are generated by the nerve cells in the brain. EEG has so many advantages such as the characteristic of the electrical recording system because it has high precision and time measurements. Another advantage is that EEG is a very inexpensive device, and can easily be operated with. Poor recording spatial resolution is one of the disadvantages of the EEG. EMOTIV provides two different headsets, which are EPOC + and Insight. Each one has different properties or features. The main differences that will serve our needs are related to the number of sensors and signal resolution. The EPOC features 16 sensors plus 2 reference sensors. Also, it provides high resolution. On the other hand, the insight has 5 sensors plus 2 reference sensors and it is less accurate than EPOC when detecting the signals (low resolution).

The second user interface is virtual reality. It is an artificial environment that is made by software and presented by the user in such a way that makes it real. Virtual reality also has so many advantages and disadvantages. One of the most important advantages is that the disabled people that are not able to experience reality can explore the virtual world, and experience the full life there. It also allows the user to experience impossible things in real life. One of the biggest drawback of the virtual reality is that people might get addicted to that virtual world which will lead them to forget their responsibilities in the real life.

The third user interface is the electromyography (EMG). It is way to access the health muscles and nerves to control and interact with it. EMG signals can be detected or transferred through a small device called electrodes. It has some advantages and disadvantages. EMG is more accurate, safe, and easy to get the wanted results. Also, EMG signals are not as complicated as the EEG when it comes to finding locations for measuring. However, 


\section{A. Roshdy et al. / Advances in Science, Technology and Engineering Systems Journal Vol. 4, No. 1, 17-26 (2019)}

noises may occur due to the distance between the user and the signals of muscles.

\subsection{Communication}

The first category to be chosen is to have wireless communication to give the user the freedom to move while using the device. The Wifi, Bluetooth, and XBEE (pronounced ZigBee) are three different types of wireless communications which varies in the Safety, range of accessibility, reliability, power consumption, and bandwidth.

Starting with WiFi, its security is lower than the other two types with 20-150 meters range of communication. Although the reliability is low it still consumes high power with its wide bandwidth. Second choice is the Bluetooth which has a bit better safety communication than the Wifi. But the main problem is the short range which is $8-30$ meters. Still it has low reliability but low power consumption with a narrow bandwidth. The third option is the ZigBee is offering a good safety communication with a good range of communication coverage 20-150 meters. It does have a high reliability compared to the other two options as well as low power consumption with a narrow bandwidth

\subsection{Decision Making and Selections}

Based on the project needs and requirements, a 3D printed design was used. The pressure sensor chosen to be LPS25MB because it is cheap and light in weight. Also MS5637-02BA03 was chosen to be the selected pressure sensor for its low cost and high accuracy. For the health sensor Pulse and Oxygen in Blood Sensor (SPO2) was chosen due to its accuracy and fast response. And the servo motor ASMC-03B was selected due to its high torque even it's a bit more expensive than other servo motors. Cobalt Li-ion battery got the highest score because of its suitable cell voltage, long life time, good energy, suitable charge time, low cost and it is safe. For the interface, EEG and EPOC allows obtaining more or variety of locations than insight. Based on the comparison, EPOC is more suitable than insight in terms of accuracy and sensors location. Finally, Arduino was chosen because it has the least price above them all. In case something happened during the coding process and the circuit got damaged we can easily buy another one because of its low price. Also, it can help us easily in our project since it has so many libraries and control system to control the exoskeleton.

\section{Results and Analysis}

The servo motors are responsible for the fingers movement according to the selected exercise. Based on the user selection the servo motors will perform the selected task. In other words, if the user chooses one the Arduino will give the corresponding signals for the motors to perform exercise one which is opening the hand five fingers then close them twice with a delay five seconds after each open or close process. The second exercise that might be selected by the user also is to open the fingers and closing them five times with the same delay time in between. The third and last exercise is opening and closing the fingers nine times. Beside this the medical sensors such as the temperature sensor is helping the assisting the user continuously check his body temperature. Another medical sensor mounted on the device is the pulse oximetry sensor to monitor the arterial oxygen saturation in the hemoglobin. Also a regular sixteen characters two lines LCD is used to show the measured and monitored data for the user with a Wi-Fi connected between the wearable device and the mobile phone application to send the collected data. Innovator X Post-Op Elbow was used as an angular controller. It provides safety to the user due to the adjustments of the angle position that we can make to prevent hand broken.

The biggest challenge of the project was to build a pattern recognition system for the brain signals to be able to detect the required movement from the brain itself with any other external method. The problem is that making a wrong pattern recognition may hurt the patient if a wrong move was done if the brain signal was not read correctly. A raw brain signal data coming from the EEG was read and analyzed by MATLAB software. MATLAB included a digital band pass and band stop filters to eliminate the noise from the brain signal.

\section{Conclusion}

Patients suffering from some kind of hand disabilities are facing a problem in their day to day activities that's why in this paper we've suggested a light weight low-cost wearable device compared to the devices used nowadays. The device designed to assist the user through two different modes of operation. The two modes are active and passive and both are helping the user to perform some hand exercises as well as monitoring his body temperature, pulses, ECG, and oxygen percentage.

Exoskeleton is a wearable device that improves the user's performance. There are two kinds of exoskeleton, which are assistive and rehabilitation. Assistive exoskeleton gives a user an extra strength to do different tasks. Rehabilitation exoskeleton reduces the recovery time and has long-term effect. This project will help many people that suffer from diseases like stroke, broken bones, spinal cord injury, hemiplegia and traumatic brain injury. Hands are so important part of the human body, so we will design a hand rehabilitation exoskeleton for stroke patients. This hand works in two modes: passive and active. It will be free size. This exoskeleton expected to increase the strength of the hand, the chance of healing, decrease the pain, the cost, and the recovery time.

Our design contains mainly four parts, first the EEG part, which will read the signals from the brain and send it to the PRU part through the Wi-Fi. PRU stands for pattern recognition unit that its function is to analyze signals. Signals were analyzed using Matlab. The third part, which is electromechanical part, will take the signals from PRU through ZigBee and control the arm servomotor using Arduino. Electromechanical part also contains five small servomotors to control the fingers to do specific exercises. Health care part contains three sensors, which are temperature sensor, pulse sensor, and ECG sensor and the results will be shown in the LCD. The user also can enter his information and choose any exercise using keypad and LCD. The last part is mobile application, which will take the results from the health care part through the Wi-Fi.

As a suggested future work and improvements the device can be improved by selecting the control mode through the brain signals with the keypad used as an extra input method. Also the keypad with the screen can be replaced by one big touch screen.

\section{Conflict of Interest}

The authors declare no conflict of interest. 


\section{A. Roshdy et al. / Advances in Science, Technology and Engineering Systems Journal Vol. 4, No. 1, 17-26 (2019)}

\section{Acknowledgment}

We would like to thank the American University of the Middle East that gave us the opportunity to carry out our research. We would also like to thank the Robotics Research center for funding the project providing us such great facilities and assistance throughout our research.

\section{References}

[1] F Sayegh, F Fadhli, F Karam, M BoAbbas, F Mahmeed, JA Korbane, S AlKork, T Beyrouthy "A wearable rehabilitation device for paralysis," 2017 2nd International Conference on Bio-engineering for Smart Technologies (BioSMART), Paris, 2017, pp.1-4.

[2] A. P. Ong and N. T. Bugtai, "Recent Developments of Robotic Exoskeletons for Hand Rehabilitation," Presented at The DLSU Research Congress 2016, pp. $1-2$.

[3] C. A. Lim 2013, "Ironman Project-Design Of Electro-Mechanical Muscle For Elbow Exoskeleton Robot," 2013.

[4] Rahman, M. A., \& Al-Jumaily, A. Design and development of a hand exoskeleton for rehabilitation following stroke. Procedia Engineering, 41, 1028-1034, 2012.

[5] Kawasaki, H., Kimura, H., Ito, S., Nishimoto, Y., \& Hayashi, H. Hand rehabilitation support system based on self-motion control, with a clinical case report. In 2006 World Automation Congress (pp. 1-6). IEEE. (2006, July).

[6] Heo, P., Gu, G. M., Lee, S. J., Rhee, K., \& Kim, J. Current hand exoskeleton technologies for rehabilitation and assistive engineering. International Journal of Precision Engineering and Manufacturing, 13(5), 807-824. (2012).

[7] Sandoval-Gonzalez, O., Jacinto-Villegas, J., Herrera-Aguilar, I., PortilloRodiguez, O., Tripicchio, P., Hernandez-Ramos, M., ... \& Avizzano, C. Design and Development of a Hand Exoskeleton Robot for Active and Passive Rehabilitation (2016).

[8] Ho, N. S. K., Tong, K. Y., Hu, X. L., Fung, K. L., Wei, X. J., Rong, W., \& Susanto, E. A. An EMG-driven exoskeleton hand robotic training device on chronic stroke subjects: task training system for stroke rehabilitation. In 2011 IEEE international conference on rehabilitation robotics(pp. 1-5). IEEE (2011, June).

[9] Babaiasl, M., Mahdioun, S. H., Jaryani, P., \& Yazdani, M. A review of technological and clinical aspects of robot-aided rehabilitation of upperextremity after stroke. Disability and Rehabilitation: Assistive Technology, 11(4), 263-280 (2016).

[10] Arata, J., Ohmoto, K., Gassert, R., Lambercy, O., Fujimoto, H., \& Wada, I. A new hand exoskeleton device for rehabilitation using a three-layered sliding spring mechanism. In Robotics and Automation (ICRA), 2013 IEEE International Conference on (pp. 3902-3907). IEEE (2013,May).

[11] Sarakoglou, I., Tsagarakis, N. G., \& Caldwell, D. G. (2004, September). Occupational and physical therapy using a hand exoskeleton based exerciser. In Intelligent Robots and Systems. (IROS 2004). Proceedings. IEEE/RSJ International Conference on (Vol. 3, pp. 2973-2978). IEEE) (2004).

[12] Capitani, S., Cremoni, A., Lindenroth, L., Secciani, N., Shaftr, A., Stilli, A., \&Venture, M. Development of low-cost portable hand exoskeleton for assistive and rehabilitation purposes. For The ENTERFACE International Workshop (pp. 1-3) (2016).

[13] W. D. Hairston, K. W. Whitaker, A. J. Ries, J. M. Vettel, J. C. Bradford, S. E. Kerick, and K. McDowell, "Usability of four commercially-oriented EEG systems," in Journal of neural engineering, 11(4), 046018, 2014.

[14] abilah Hamzah, Haryanti Norhazman, Norliza Zaini and Maizura Sani. Classification of Eeg Signals Based on Different Motor Movement Using Multi-layer Perceptron Artificial Neural Network. Journal of Biological Sciences, 16: 265-271 (2016).

[15] F. Lotte, L. Bougrain, and M. Clerc, "Electroencephalography (EEG)-based Brain Computer Interfaces," Wiley Encyclopedia of Electrical and Electronics Engineering, Wiley, pp. 44, 2015.

[16] F. Lotte, "A Tutorial on EEG Signal Processing Techniques for Mental State Recognition in Brain-Computer Interfaces," Eduardo Reck Miranda; Julien Castet. Guide to Brain-Computer Music Interfacing, Springer, 2014.

[17] M. M. Moazzami, "EEG signal Processing in Brain-Computer Interface" (Doctoral dissertation, Michigan State University), 2012.

[18] S AlAwadhi, N AlHabib, D Murad, F AlDeei, M AlHouti, T Beyrouthy, S Al-Kork "Virtual reality application for interactive and informative learning," 2017 2nd International Conference on Bio-engineering for Smart Technologies (BioSMART), Paris, 2017, pp. 1-4.
[19] S.A. Awadhi, N.A. Habib, D. Al-Murad, F.A. deei, M.A. Houti, T. Beyrouthy, S. Al-Kork "Interactive Virtual Reality Educational Application", Advances in Science, Technology and Engineering Systems Journal, vol. 3, no. 4, pp. 72-82 (2018).

[20] Reaz, M. B. I., Hussain, M. S., \& Mohd-Yasin, F. Techniques of EMG signal analysis: detection, processing, classification and applications. Biological procedures online, 8(1), 11-35 (2006).

[21] S. Said, S. AlKork, T. Beyrouthy and M. F. Abdrabbo, "Wearable biosensors bracelet for driver as health emergency detection," 2017 2nd International Conference on Bio-engineering for Smart Technologies (BioSmart), Paris, 2017, pp. 1-4.

[22] Hasan S., Al-Kandari K., Al-Awadhi E., Jaafar A., Al-Farhan B., Hassan M., Said S. and AlKork S. (2018). Wearable Mind Thoughts Controlled Open Source 3D Printed Arm with Embedded Sensor Feedback System.In Proceedings of the 2nd International Conference on Computer-Human Interaction Research and Applications - Volume 1: CHIRA, ISBN 978-989758-328-5, pages 141-149.

[23] S. K. A. Kork, I. Gowthami, X. Savatier, T. Beyrouthy, J. A. Korbane and S. Roshdi, "Biometric database for human gait recognition using wearable sensors and a smartphone," 2017 2nd International Conference on Bioengineering for Smart Technologies (BioSMART), Paris, 2017, pp. 1-4.

[24] D. Planelles, E. Hortal, Á. Costa, A. Ubeda, E. Iaez, and J. M. Azorin, "Evaluating classifiers to detect arm movement intention from eeg signals," Sensors, 14(10), pp. 18172-18186, 2014.

[25] Taha Beyrouthy, Samer Al. Kork, J. Korbane, M. Abouelela, "EEG Mind Controlled Smart Prosthetic Arm - A Comprehensive Study", Advances in Science, Technology and Engineering Systems Journal, vol. 2, no. 3, pp. 891899 (2017).

[26] T. Beyrouthy, S. K. Al Kork, J. A. Korbane and A. Abdulmonem, "EEG Mind controlled Smart Prosthetic Arm," 2016 IEEE International Conference on Emerging Technologies and Innovative Business Practices for the Transformation of Societies (EmergiTech), Balaclava, 2016, pp. 404409. 